\title{
SWI/SNF Complex Subunit SMARCC2
}

National Cancer Institute

\section{Source}

National Cancer Institute. SWIISNF Complex Subunit SMARCC2. NCI Thesaurus. Code C19839.

SWI/SNF complex subunit SMARCC2 (1214 aa, 133 kDa) is encoded by the human SMARCC2 gene. This protein plays a role in both transcriptional regulation and nucleosome remodeling. 